\title{
AN ARTIFICIAL LIFE MODEL FOR IMAGE ENHANCEMENT
}

\author{
Alex F. de Araujo ${ }^{(*)}$, João Manuel R.S. Tavares
}

Instituto de Engenharia Mecânica e Gestão Industrial, Faculdade de Engenharia, Universidade do Porto, Portugal

${ }^{(*)}$ Email: fa.alex@gmail.com

\begin{abstract}
This paper presents an artificial life model for image enhancement. The results of some traditional enhancement methods were analyzed and compared with the ones obtained by the model proposed. The qualitative and quantitative tests performed allowed to conclude that the new model is promising, as is able to enhance transitions of the objects presented in the original images and make them more visually perceptible.
\end{abstract}

\section{INTRODUCTION}

There are several factors that can contribute to damage the information in images, such as loss of focus, presence of noise, reflections and shadows, and insufficient illumination. Image enhancement methods have been developed to reduce the effect of such damages, by improving the contrast between the objects represented, emphasizing their more significant features (Hashemi, 2010). Artificial models, inspired on the biological processes that characterize living organisms, have been adopted to perform computational image analysis tasks (Hamarneh, 2009), (McInerney, 2002). Such biological processes include growing, natural selection, evolution, locomotion and learning (Terzopoulos, 1999).

This paper presents a new artificial life model, which is inspired on the behavior of an herbivore organism when it is in an environment and selects its food, for image enhancement. Thus, considering an environment containing herbs of different heights, the smaller herbs are eaten first, because they are smoother and more nutritional. Therefore, there will be a tendency to increase the differences between the shorter and taller herbs due to the motion and eating process of the organism, in a similar way as it is desired in the image enhancement.

Qualitative and quantitative comparisons performed on the results obtained by the proposed model and some image enhancement traditionally methods allowed to conclude that our solution is promising, being able to improve the quality of the damaged images and their visual perception considerably.

\section{RESULTS AND CONCLUSIONS}

The PSNR (Peak Signal Noise Ratio) indices calculated from the comparison of the original images and ones obtained by the proposed model and some image enhancement traditionally methods are represented in Figure 1. This figure allows to realize that the adopted model returned images with the best indices. The image test set used was composed by syntactic images created using an image editor and the well-known "Lena" and "Cameramen" images, both of them with the contrast affected by the addition of controlled noise and blurring. Regarding the "Lena image", Figure 2 depicts the enhancement results, and Table 1 presents the associated PSNR indices.

This work has shown that the proposed artificial life model for image enhancement is promising, leading to better results than the enhancement traditionally methods. To improve 
the efficiency of our model, we intend to develop an enhanced cognitive system, and apply optimization techniques and parallel programming to speed up the computational process.

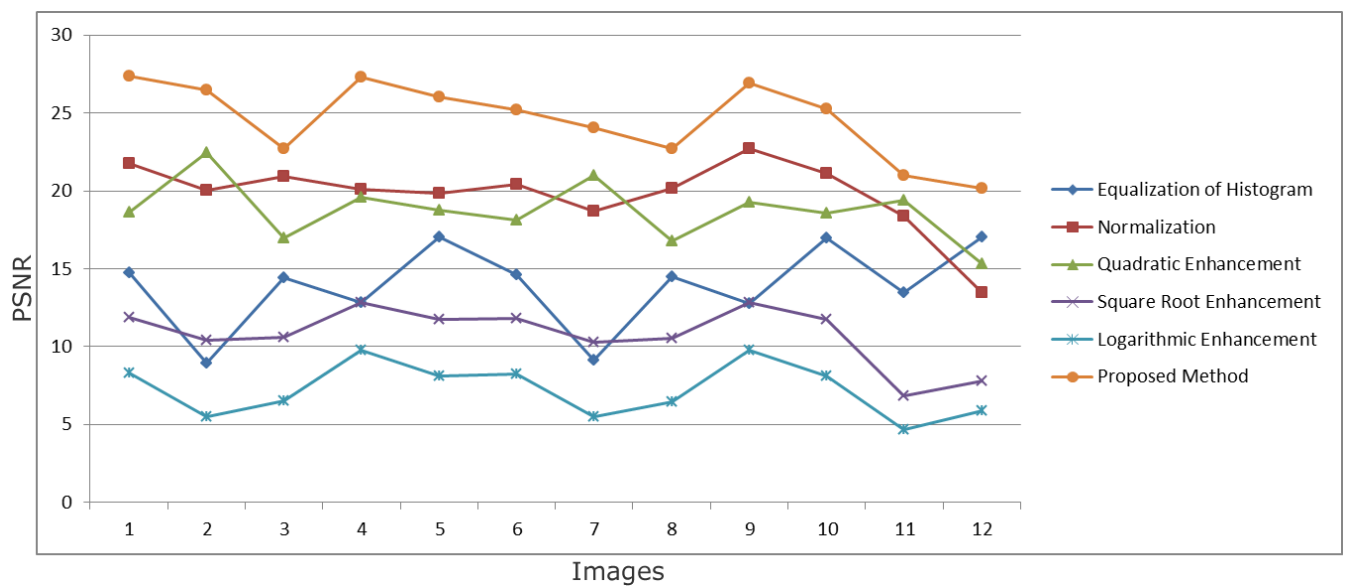

Figure 1 - Graph with the PSNR indices for the images tested.

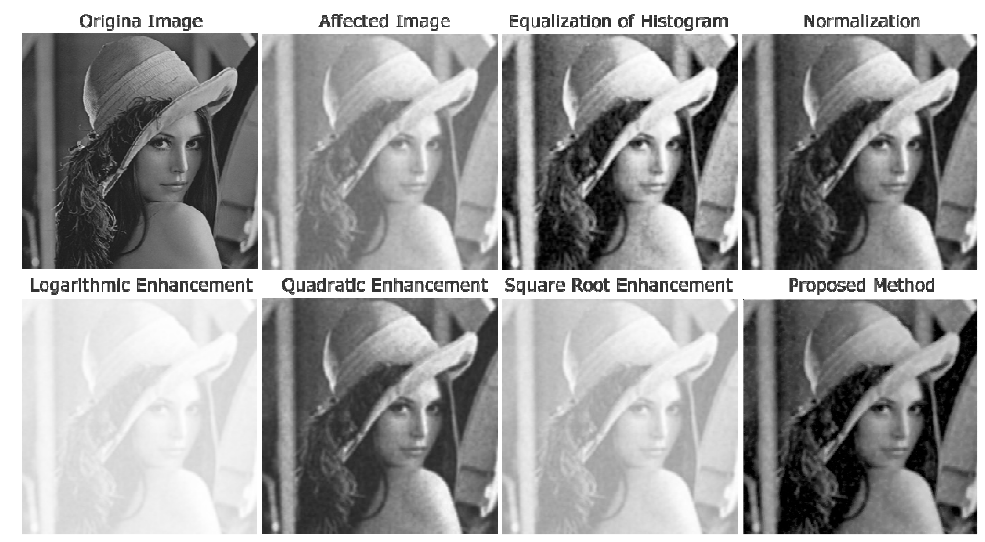

Figure 2 - Results of the enhancement methods applied to "Lena image".
Table 1- PSNR of "Lena" image.

\begin{tabular}{|c|c|}
\hline METHOD & PSNR \\
\hline $\begin{array}{c}\text { Equalization } \\
\text { of Histogram }\end{array}$ & 13.49 \\
\hline Normalization & 18.36 \\
\hline $\begin{array}{c}\text { Quadratic } \\
\text { Enhancement }\end{array}$ & 19.43 \\
\hline $\begin{array}{c}\text { Square Root } \\
\text { Enhancement }\end{array}$ & 6.84 \\
\hline $\begin{array}{c}\text { Logarithmic } \\
\text { Enhancement }\end{array}$ & 4.70 \\
\hline $\begin{array}{c}\text { Proposed } \\
\text { Method }\end{array}$ & 20.99 \\
\hline
\end{tabular}

\section{ACKNOWLEDGMENTS}

This work was partially done in the scope of the projects with references PTDC/EEACRO/103320/2008, UTAustin/MAT/0009/2008 and UTAustin/CA/0047/2008, financially supported by FCT - Fundação para a Ciência e a Tecnologia in Portugal. The first author would like to thank his PhD grant from FCT with reference SFRH/BD/61983/2009.

\section{REFERENCES}

[1]-Hamarneh G, McIntosh C, McInerney T, Terzopoulos D. Deformable Organisms: An Artificial Life Framework for Automated Medical Image Analysis. Book: Computational Intelligence In Medical Imaging: Techniques and Applications, 2009, p. 433-474.

[2]-Hashemi S, Kiani S, Noroozi N, Moghaddam ME. An image contrast enhancement method based on genetic algorithm. In Pattern Recognition Letters. 2010, Vol. 31:13, p. 1816-1824.

[3]-McInerney T, Hamarneh G, Shenton M, Terzopoulos D. Deformable organisms for automatic medical image analysis. In Medical Image Analysis. 2002, Vol. 6:3, p. 251-266.

[4]-Terzopoulos D. Artificial life for computer graphics. In Communications of the ACM, 1999, Vol. 42:8, p. 32-42. 